\title{
Les textes en ligne de présentation d'universités
}

Du texte descriptif au discours idéologique

Jeoffrey Gaspard

\section{(2) OpenEdition}

Journals

Édition électronique

URL : http://journals.openedition.org/communicationorganisation/4415

DOI : 10.4000/communicationorganisation. 4415

ISSN : $1775-3546$

Éditeur

Presses universitaires de Bordeaux

Édition imprimée

Date de publication : 1 décembre 2013

Pagination : 189-202

ISBN : 978-2-86781-878-3

ISSN : $1168-5549$

\section{Référence électronique}

Jeoffrey Gaspard, «Les textes en ligne de présentation d'universités », Communication et organisation

[En ligne], 44 | 2013, mis en ligne le 06 juin 2014, consulté le 19 avril 2019. URL : http://

journals.openedition.org/communicationorganisation/4415; DOI : 10.4000/

communicationorganisation. 4415 


\title{
Les textes en ligne de présentation d'universités du texte descriptif au discours idéologique
}

\author{
Jeoffrẹ̣ Gaspard'
}

Nous inscrivant dans la lignée des travaux de l'École française d'analyse du discours, nous admettons qu'il existe, en parallèle à l'ordre de la langue régissant la construction de phrases, un ordre du discours régissant la construction d'énoncés. Plus souples mais non moins prégnantes que celles de la grammaire, les contraintes relatives au discours produisent également des régularités, observables en corpus, que l'analyste, dans un premier temps, se proposera de décrire. Si les « faits de discours (...) s'appuient nécessairement sur des faits de langue » (Krieg-Planque, 2007 : 66), ces régularités discursives tirent en outre leur origine de contraintes contextuelles ${ }^{2}$, de sorte que la notion de discours « implique l'articulation du linguistique et du social, du discursif et de l'interdiscursif, et la prise en compte des contraintes génériques et institutionnelles gouvernant le discours » (Amossy \& Herschberg-Pierrot, 2007 : 106). Non directement assimilé à la parole individuelle en raison du caractère distribué de son émergence, le discours est alors entendu comme « une dispersion de textes que leur mode d'inscription historique permet de définir comme un espace de régularités énonciatives " (Maingueneau, 1984 : 5). L'analyse du discours ${ }^{3}$ s'écarte ainsi, tout en s'en émancipant, de la dichotomie langue / parole instaurée par Saussure, puisque « la parole n'est ni l'objet de la linguistique ni celui de l'analyse du discours, ces deux-ci étant soucieuses de délimiter et de décrire des régularités observables chez tous les locuteurs ainsi que dans les différentes situations de communication » (Sarfati, 2005 : 15). Par ailleurs, et dans un second temps, l'analyste tentera de

1 Centre de recherche ReSIC, Université Libre de Bruxelles (ULB) ; jgaspard@ulb.ac.be

$2 \mathrm{Si}$ «la linguistique s’intéresse aux propriétés du langage qui demeurent intactes lorsqu'on déconnecte un énoncé des conditions singulières de son énonciation » (Milner, 1995 : 118), l'analyse du discours considérera que ces " conditions singulières ", contextuelles, si plus ou moins identiques à travers des situations de communication plus ou moins analogues, génèreront également des " constantes ", en tant que "formes de combinaisons de formes » (Bakhtine, 1984 : 287) récurrentes. Toutefois, les facteurs propres aux situations de communications étant singulièrement complexes, ces constantes ne seront jamais des règles mais plutôt des régularités (patterns en anglais) d'ordre éventuellement statistique.

3 Nous préférons le syntagme analyse du discours à celui de linguistique du discours afin d'insister sur le fait que l'analyste du discours « ne peut être purement linguiste : le linguiste en tant que tel s'intéresse à une linguistique, éventuellement du discours, non au discours en tant que tel» (Achard, $1986: 16$ ). 
faire correspondre la sélection et la combinaison des formes aux circonstances de la situation de communication ayant présidé à leur choix et surdéterminé la matérialité discursive d'énoncés toujours socio-historiquement situés ; une analyse du discours visera donc la description et l'explication de pratiques discursives, qui définissent « à une époque donnée, et pour une aire sociale, économique, géographique ou linguistique donnée, les conditions d'exercice de la fonction énonciative » (Foucault, $2010: 162$ ).

En adoptant ces outils conceptuels, nous proposons, à travers cette contribution, d'explorer les textes ${ }^{4}$ en ligne de présentation d'universités. Nous désirons montrer que la pratique discursive consistant à promouvoir en ligne ce type d'institutions génère des textes (numériques) à partir desquels il est possible de dégager de ces régularités ${ }^{5}$ qui témoignent de la présence d'un ordre du discours particulier.

\section{Le tournant communicationnel des universités}

Il n'est besoin d'être professeur ou étudiant pour mesurer l'étendue grandissante des pratiques de communication au sein des universités : n'importe quel lecteur de journaux peut, d'une lecture à l'autre, se trouver exposé à bon nombre de publicités visant à informer tel étudiant de la tenue de journées portes-ouvertes ou tel autre bailleur de fonds potentiel de la création d'une fondation. Pour Defays (2009 : 13), en effet, « l'université-tour d'ivoire, qui pratiquait des discours réservés aux initiés, a laissé la place à l'université médiatique, qui communique, qui rayonne, qui séduit ». Trois facteurs peuvent expliquer l'importance croissante des activités de communication, tant internes qu'externes (Engwall, 2008) : premièrement, un phénomène de concurrence interinstitutionnelle exacerbée, qui requiert une visibilité optimale des établissements et de leurs activités ; deuxièmement, un processus de socialisation, suivant lequel le personnel administratif mais également scientifique adoptent une attitude plus conciliante envers des activités de communication autrefois dénigrées ; et enfin troisièmement, une tendance au mimétisme interinstitutionnel mais également interprofessionnel, les responsables de communication s'échangeant désormais de bonnes pratiques ${ }^{6}$. L'expansion de ces activités, en termes de ressources humaines, financières

\footnotetext{
4 Le corpus sur lequel nous nous basons est constitué de textes obtenus à partir des pages de présentation institutionnelle (voir ci-dessous) de 80 sites web d'universités francophones en Europe (France, Suisse romande, Belgique francophone et Luxembourg). Les sites web ont été consultés les 3 et 4 novembre 2012. Selon AnaText, le corpus est composé de 66541 occurrences (hors ponctuation) et 7416 formes graphiques.

5 En vue d'opérer notre travail de description des régularités observées, nous avons commencé par nous familiariser avec le corpus, en effectuant des lectures linéaires de plusieurs de ses parties. Les régularités repérées dans ce premier temps ont ensuite été corroborées par les outils textométriques et approfondies par l'analyse, ce qui débouche sur ces premiers résultats exploratoires. Pour les présentes analyses, le corpus a été traité par les logiciels d'analyse textométrique TXM (version 0.7) et AnaText (2.0) (en ligne), le correcteur orthographique et grammatical Antidote (version 6.1) et enfin par le logiciel d'analyse sémantique Tropes (version 8.3).

6 L'association EUPRIO (European Universities Public Relations E Informations Officers) constitue par exemple un terrain d'échanges et de rencontres propice à cette fin.
} 
et techniques déployées, leur professionnalisation, en termes de diplômes, compétences et recrutement du personnel leur étant attachés, et enfin leur valorisation, caractérisent ainsi un tournant communicationnel au sein des universités (Engwall, 2008).

Si cette communication institutionnelle se décline en de nombreux artefacts ${ }^{7}$, elle se pratique aujourd'hui prioritairement en ligne, les technologies de la communication occupant désormais une place centrale dans les dispositifs promotionnels. De tous les outils techno-discursifs ${ }^{8}$ à disposition, le site web universitaire", "vitrine institutionnelle " (Barats, 2009) pour tout individu désirant obtenir des informations relatives à l'institution, constitue un instrument relationnel et promotionnel incontournable ${ }^{10}$. Parmi les différents modules ${ }^{11}$ que propose généralement le site web, celui relatif à la présentation de l'institution - souvent intitulé $\grave{A}$ propos de l'université ou Université est sans doute celui qui correspond le plus à cette visée de promotion de l'établissement dans son ensemble. Les textes numériques qui s'y trouvent disséminés constituent donc des productions discursives emblématiques du discours promotionnel universitaire que nous souhaitons explorer.

\section{Les textes en ligne de présentation d'université : types et genres de discours}

Un des apports les plus significatifs de l'analyse du discours est d'avoir étendu l'étude des types et genres de discours au-delà du champ littéraire en ayant montré que les productions discursives, en société, pouvaient être également typologisées selon différents critères. Ainsi, ces textes de présentation peuvent être catégorisés suivant trois critères au moins : leur origine situationnelle (1), leur fonction communicationnelle (2) et leur support matériel (3). Concernant leur origine situationnelle, il suffira de préciser que ces textes, étant produits par des scripteurs membres de services de communication sous la responsabilité énonciative des autorités de l'université, produisent, ce faisant, un « discours autorisé dans un milieu donné », ce que Oger \& OllivierYaniv (2003) estiment être la marque des discours institutionnels. Du point

7 Voir, par exemple, Askehave (2007) et Osman (2008), qui ont tous deux traité des brochures universitaires.

8 Le terme signifie ici que communiquer en ligne est une pratique à la fois discursive (dont le substrat est l'« outil» langagier) mais également technique (« outil » informatique). Les contraintes, constitutives de cette interaction discours/technique, font émerger des configurations (hyper)textuelles précises qui se trouvent être en partie caractéristiques de l'hypergenre « site web institutionnel» (voir plus bas).

9 Nous proposons de circonscrire le « site web universitaire » par un critère purement technique : est considéré comme tel l'ensemble des pages web appartenant à un même nom de domaine (par exemple, les pages reprises sous unistra.fr pour le domaine de l'Université de Strasbourg), auquel s'ajoute un critère fonctionnel : est considéré comme tel un site dont la visée est de 1) promouvoir et valoriser une institution ou organisation, 2) informer des publics au sujet de cette institution ou organisation et enfin 3) fournir des services en ligne propres à cette institution ou organisation (e.g. cours et inscription en ligne, dans le cas des universités).

10 Sur l'importance des sites web institutionnels pour les universités, voir Saichaie (2011).

11 On retrouve ainsi systématiquement des modules de présentation des formations, de la recherche, du caractère international de l'institution et de la vie étudiante, mais également des pages consacrées aux relations avec les entreprises et à l'insertion professionnelle des diplômés, à la documentation/bibliothèques, aux actualités, aux ressources humaines/recrutement ou encore des pages destinées aux relations médias/presse (Gaspard, 2013a). 
de vue communicationnel, ces textes appartiennent, dans le même temps, aux discours de type promotionnel ${ }^{12}$, puisqu'ils ont pour objectifs de présenter et de promouvoir l'institution universitaire qu'ils mettent en scène. Enfin, concernant leur matérialité, ces (hyper)textes sont également numériques de sorte qu'une partie de leurs caractéristiques peut être expliquée par les contraintes spécifiques que génère une écriture en ligne ${ }^{13}$.

Ensuite, l'appartenance de ces textes à un genre de discours déterminé, coextensifà l'activité de communication qui les a fait naître, devrait contraindre, en partie du moins, ce qui doit être dit et comment cela doit être dit, puisque « des discours produits dans des conditions sociales identiques présenteront des affinités formelles suffisamment fortes pour que l'on puisse considérer qu'ils relèvent du même genre » (Le Bart \& Teillet, 2004 : 53). Or, bon nombre d'«affinités formelles » décrites ci-dessous se retrouvent également dans les textes de présentation de musées, d'organisations non gouvernementales ou d'institutions publiques diverses, qui, dans un environnement devenu concurrentiel, ont également besoin de s'auto-promouvoir ; cet isomorphisme suggère donc une actualisation d'un genre de discours particulier.

Saichaie (2011) relevait l'importance de la légitimité institutionnelle pour expliquer les similarités flagrantes observées entre textes promotionnels et la façon dont les scripteurs scrutent le discours promotionnel des autres institutions :

DiMaggio and Powell's concept of mimetic isomorphism provides insight into why organizations utilize similar discourse when promoting their institutions. Mimetic isomorphism suggests that institutions adopt the practices of organizations they perceive to be of higher standing and prestige. (Saichaie, 2011 : 174)

Cette crainte d'une perte de légitimité ${ }^{14}$, équivalente dans d'autres institutions publiques, pourrait expliquer que les scripteurs rechignent à se distancer, en discours, de normes discursives prévalentes, en particulier celles

12 Avec Fevre-Pernet (2009), nous sommes d'avis que le discours de type promotionnel s'oppose au discours de type publicitaire en ce sens que les genres du premier type « ont un caractère clairement/explicitement incitatif (faire acheter) alors que les genres du champ publicitaire cherchent en général à masquer par des scénographies souvent très travaillées pouvant d'ailleurs emprunter à d'autres genres (récit, fiction, témoignage, farce, sketch...) ». Bien entendu, dans le cadre d'une communication institutionnelle, il ne s'agira pas de "faire acheter » mais plutôt de "faire adhérer », et le " produit » considéré est un objet sémiotique intangible : l'université $x$.

13 Par exemple, les textes se doivent d'être adaptés à une lecture sur écran : bien que le web permette, en théorie, des espaces de stockage infinis, en pratique, la longueur des textes numériques gagne en lisibilité à être minimale. Ce faisant, la grande majorité des textes analysés comprennent une extension descriptive limitée, celle-ci étant dépendante « à la fois de la quantité d'information à transmettre, du support matériel qui lui sert de vecteur et du type de destinataire à qui elle doit être adressée » (Charaudeau, 1992: 699).

14 Dans le même ordre d'idées, Morphew \& Hartley (2006), dans le cas des « mission statements ", écrivent : "Institutional theorists point to organizational artifacts like mission statements and knowingly describe them as ritualistic or mythological. From this point of view, mission statements are certainly important but not for the direction they provide. Rather, they serve a legitimating function. Mission statements are valuable because they - and the elements within them - show that the organization in question understands the 'rules of the game' ". 
émanant d'universités jugées prestigieuses. L’isomorphisme institutionnel se dédouble alors d'un isomorphisme discursif.

Dans le cas du web, toutefois, l'approche du genre se complexifie. Maingueneau (2013) suggère ainsi que « le web transforme (...) les conditions de la communication, la manière dont on peut envisager le genre et la notion même de textualité ». En effet, étant donné que « les sites (...) sont soumis à un ensemble de contraintes techniques, et [qu'une] homogénéisation est renforcée par la nécessité de pouvoir circuler par hyperliens d'un site à l'autre », il estime que «c'est le couple hypergenre / scénographie qui joue [un] rôle central dans la textualité navigante ${ }^{15}$ ». En somme, le web proposerait quelques grands « hypergenres ", tels que le blog, le site d'information, le site institutionnel, etc., qui mobiliseraient des scénographies ${ }^{16}$ variées. En fin de compte, nous suggérons donc que ces textes de présentation institutionnelle relèvent d'un sous-genre de l'hypergenre site institutionnel, qui présente certaines caractéristiques, les plus marquantes étant résumées ci-dessous.

\section{Des textes qui promeuvent en décrivant}

Fondamentalement, la fonction de ces textes consiste à promouvoir l'institution universitaire en la décrivant d'une manière valorisante, dans le but de "faire adhérer ${ }^{17}$. Dans ce cas, la description équivaut à une argumentation, à l'instar des nombreuses descriptions de produits que génère le discours publicitaire, pour lequel « la persuasion est très souvent déguisée en description » (Adam \& Bonhomme, 2012 : 125). Ces textes construisent ainsi, verbalement ${ }^{18}$, un objet sémiotique intangible (l'université $x$ ) qu'il s'agit de caractériser, et dont la représentation, à travers la procédure descriptive, se veut résolument positive. L'exemple suivant reprend une séquence descriptive caractéristique :

1. Considérée comme la $1^{\text {re }}$ université juridique française, l'Université Panthéon-Assas défend la diversité et cultive une vraie synergie entre recherche, enseignement et application. (...) Elle développe des partenariats avec les principaux cabinets ou entreprises industrielles et financières en France, en Europe et dans le monde. Rassemblant les compétences d'enseignants renommés et internationalement connus, elle peut s'enorgueillir

15 L'auteur distingue trois types de textualité : 1) la textualité conversationnelle (orale), 2) la textualité planifiée (qui peut être soit orale - dialogale ou monologale - soit écrite - linéaire ou tabulaire - et enfin 3) la textualité navigante, propre au web.

16 Selon Maingueneau (2013), les scénographies en ligne autorisent deux niveaux d'analyse : le niveau strictement verbal et le niveau numérique, qui comprend lui-même les niveaux iconotextuel (images et textes), architectural (structuration du site) et procédural (mode de navigation et instructions). Dans le présent travail, nous proposons de nous concentrer uniquement sur le niveau verbal.

17 Contrairement aux textes publicitaires traditionnels dont l'objectif est de « faire acheter ».

18 Mais pas uniquement : les textes sont généralement accompagnés d’un matériau sémiotique (audio-)visuel (photos, vidéos, logo, etc.) dans le paratexte. 
d'une production scientifique d'une extrême richesse (texte de l'Université Panthéon-Assas).

L'importance du descriptif est suggérée par le diagnostic de Tropes, suivant lequel ces textes manifestent un "style plutôt descriptif ${ }^{19}$ ", comptabilisent $63 \%$ de verbes factifs ${ }^{20}$. Mais d'autres indices viennent étayer leur caractère descriptif. Ainsi, l'usage du présent de l'indicatif ${ }^{21}$ est marquant : AnaText comptabilise 2626 occurrences de cette forme, ce qui correspond à plus de la moitié $(55 \%)$ de l'ensemble des formes verbales détectées. Ici, l'indicatif présent est au service de l'objectif communicationnel visé, c'est-à-dire caractériser les établissements tels qu'ils se présentent hic et nunc, dans la situation d'énonciation, en leur affectant des attributs, qualités, etc., que l'on voudrait pérennes. On retrouve également bon nombre d'appositions (tant nominales, adjectivales que verbales) et de constructions pronominales qui, dans le cas de ces dernières, mobilisent fréquemment l'anaphore elle, un des lexèmes les plus fréquents du corpus, pour éviter de répéter la dénomination de l'université dont il est question. Notons également l'absence marquée, dans la plupart des textes, du pronom personnel nous, qui dénote une tentative d'effacement des locuteurs (employés ou autorités de l'université) au profit d'un énonciateur impersonnel omniscient. Aussi, la structure phrastique d'un énoncé typique se présente comme suit :

$([$ apposition $]),([$ déterminant ou pronom possessif $])\left[\right.$ noyau $=\left\{\begin{array}{c}\text { "université" } \\ \text { "elle" } \\ \text { nom propre }\end{array}\right](,[$ apposition $]),[$ verbe 3ème pers.sg.ind.prés. $][$ prédicat $]$.

Voici, ci-dessous, une occurrence représentative de cette structure :

2. Soucieuse d'apporter à ses étudiants une solide formation de base et une initiation à la recherche et à la démarche critique, l'université sait aussi qu'il faut rencontrer les besoins nouveaux de la société et de la science par une offre innovante et adaptée à de nouveaux publics (texte de l'Université libre de Bruxelles).

Nous suggérons que l'emploi de ce type de structure, dans le cadre de descriptions, n'est pas anodin ; en tant que fait phraséologique, cette structure est au service de l'intention communicationnelle visée : chacun des éléments (apposition, verbe, complément d'objet) peut être le support d'une information descriptive.

$\mathrm{Au}$-delà de ces aspects formels, le corpus comprend également des régularités proprement discursives, autant de (combinaisons de) formes qui se trouvent être récurrentes dans l'intradiscours : ces termes et segments répétés, plus ou moins figés, témoignent de routines et stéréotypies verbales privilégiées

19 À côté des styles argumentatif, narratif et énonciatif, le style descriptif, selon le manuel du logiciel, désigne le cas où « un narrateur décrit, identifie ou classifie quelque chose ou quelqu'un ».

20 Toujours selon le manuel, les verbes factifs, à côté des verbes dits statifs, déclaratifs et performatifs, « expriment des actions ("travailler", "marcher",...) ».

21 À la $3^{\mathrm{e}}$ personne du singulier. 
dans l'économie du discours promotionnel. Le Tableau 1 reprend par exemple les 10 premiers verbes et adverbes ${ }^{22}$ (lemmatisés) du corpus, accompagnés de leur fréquence. Ces différentes formes, répétées, sont mobilisées pour constituer éventuellement des co-occurrences, elles-mêmes récurrentes, dont la sémantique s'accorde également avec l'objectif communicationnel visé, c'est-à-dire promouvoir en décrivant.

\begin{tabular}{|c|c|c|c|}
\hline Verbes & $\mathrm{n}$ & Adverbes & $\mathrm{n}$ \\
\hline être & 941 & plus & 300 \\
avoir & 275 & aussi & 96 \\
proposer & 113 & également & 83 \\
développer & 99 & ainsi & 77 \\
faire & 84 & notamment & 72 \\
permettre & 78 & tout & 60 \\
mettre & 76 & près & 54 \\
offrir & 72 & très & 53 \\
inscrire & 67 & aujourd'hui & 53 \\
créer & 58 & ne & 45 \\
\hline
\end{tabular}

Tableau 1. Dix premiers verbes et adverbes (lemmatisés) les plus fréquents

Ainsi, le verbe le plus fréquemment employé est être, qui se combine le plus souvent, à l'instar d'avoir, avec la forme université, qui est son co-occurrent le plus statistiquement significatif. Le syntagme l'université $[x]$ est constitue donc un noyau régulier des énoncés promotionnels étudiés. Ces deux verbes fréquemment répétés, avec proposer, permettre et offrir, sont bel et bien employés dans une optique descriptive pour attribuer des qualités, propriétés, etc. à l'université. Quant aux adverbes aussi, également, et notamment, avec la conjonction et, fréquents dans le corpus, ils sont voués à marquer l'addition d'éléments constitutifs de l'objet décrit.

Sans surprise, les descriptions sont également caractérisées par une valorisation systématique des aspects traités, repérée entre autres par la sélection d'adjectifs à connotation positive : grand, fort, nombreux, haut, reconnu, majeur, prestigieux, etc. Voici, ci-dessous, un énoncé actualisant à la fois trois de ces adjectifs :

3. L'Université Bordeaux 1 affiche de solides références internationales qui se manifestent par l'afflux d'un grand nombre d'étudiants et de chercheurs étrangers ainsi que par un fort courant d'échanges scientifiques qui la relie à des universités et laboratoires prestigieux situés dans toutes les régions du monde (texte de l'Université de Bordeaux 1).

Notons enfin l'usage fréquent de l'énumération (4), sous la forme d'une simple liste, qui permet une présentation synthétique d'informations factuelles, et l'emploi de slogans (5). 


\section{L'UJF compte ainsi :}

- 5 académiciens des sciences;

- 53 enseignants-chercheurs membres de l'Institut universitaire de France;

- 6 médailles d'argent CNRS et 2 médailles de bronze CNRS;

- 1 prix Turing obtenu en 2007 par Joseph Sifakis (...), (texte de l'Université de Grenoble Joseph Fourier - Grenoble).

5. Nous multiplions les talents (Université de Montpellier 1). Multilingual. Personalised. Connected (Université du Luxembourg) Votre avenir commence ici (Université de Nice - Sophia Antipolis).

En somme, ces textes rappellent en partie les traditionnelles brochures papier, dont la forme n'a pas été fondamentalement altérée par une transposition en ligne.

\section{Des textes sous influence}

En plus de ces régularités dont nous pouvons penser qu'elles sont transverses aux différents genres promotionnels (usage du présent, appositions, adjectifs évaluatifs positifs, énumérations, etc.), les textes comprennent également des régularités qui s'apparentent à la formation discursive ${ }^{23}$ à laquelle ils s'intègrent. Dans un travail antérieur, nous suggérions en effet qu'une «standardisation [du discours promotionnel], en actualisant des "éléments de langage" identiques et en valorisant des atouts (réels ou, le plus souvent, escomptés) similaires à chaque institution (internationalisation de l'enseignement et de la recherche, professionnalisation des chercheurs, adéquation entre compétences et marché du travail, évaluations des activités universitaires, etc.), témoigne d'une surdétermination du discours par [un] contexte européen englobant » (Gaspard, 2013a). Justifié notamment par le processus de Bologne et la stratégie de Lisbonne, ce contexte, commun à l'ensemble des universités francophones (publiques) en Europe et caractérisé par une mise en concurrence entre institutions (voir Bruno, Clément et al, 2010), constitue bien un système de contraintes sémantiques ${ }^{24}$ auquel les textes semblent se conformer. Ainsi, certaines formules caractéristiques et termes répétés, mais aussi les types

23 Pour rappel, la formation discursive, idéologique, est « le lieu d'articulation de la langue et du discours qui détermine 'ce qui peut et doit être dit par un sujet' dans une situation de communication donnée » (Darbellay, 2005 : 240) et avec laquelle doivent se conformer les énoncés produits. Ainsi, « considérer le discours comme un système de règles historiquement déterminées et l'inscrire dans une formation discursive institutionnellement définie, c'est prendre acte du fait que parler c'est [toujours] 'parler différemment tout en parlant la même langue'» (ibidem). (Foucault, 2010 : 161) précisait que si «la régularité d'une phrase est définie par les lois d'une langue, et celle d'une proposition par les lois d'une logique, la régularité des énoncés est définie par la formation discursive elle-même ».

24 Un tel système, selon Maingueneau, « ne vise nullement à engendrer des phrases grammaticales mais à définir des opérateurs d'individuation [d'un discours], un filtre qui fixe les critères en vertu desquels certains textes se distinguent de l'ensemble des textes possibles comme appartenant à une formation discursive déterminée " (Maingueneau, 1984 : 45). Un système de contraintes sémantiques « n’engendre pas des énoncés mais constitue seulement un filtre d'énoncés » et régit « la conformité des énoncés réalisés aux contraintes de leur formation discursive » (ibidem : 72). 
de destinataires ciblés dans les formules d'adresse en pages d'accueil, par exemple, entrent en résonance avec les textes constitutifs d'un interdiscours européen sur l'enseignement supérieur et la recherche ${ }^{25}$, ce que trahissent les relations intertextuelles invariablement tissées avec cette formation discursive (néolibérale). Le Tableau 2 reprend par exemple les 30 premières formes graphiques les plus spécifiques ${ }^{26} \mathrm{du}$ corpus.

\begin{tabular}{|l|l|}
\hline \multicolumn{2}{|c|}{ Formes graphiques } \\
\hline 1. partenariat & 16. science \\
2. insertion & 17. offre \\
3. campus & 18. professionnel \\
4. formation & 19. continu \\
5. enseignant & 20. laboratoire \\
6. universitaire & 21. excellence \\
7. environnement & 22. domaine \\
8. scientifique & 23. programme \\
9. institut & 24. niveau \\
10. enseignement & 25. culture \\
11. chercheur & 26. entreprise \\
12. site & 27 mission \\
13. culturel & 28 cadre \\
14. recherche & 29 social \\
15. international & 30 projet \\
\hline
\end{tabular}

Tableau 2. Formes graphiques (lemmatisées) les plus spécifiques

L'analyse de ces spécificités confirme la prédominance de termes typiques mais peu surprenants - campus, universitaire, scientifique, institut, chercheur, etc. sont escomptés dans des textes traitant d'universités. Toutefois, d'autres formes attirent davantage l'attention - partenariat, insertion, formation, international, offre, professionnel, continue, excellence, niveau, et entreprise - étant donné qu'elles sont justement celles mobilisées par la formation discursive dont nous parlons. Certaines de ces formes se combinent pour former des segments répétés, que l'on retrouve également dans l'interdiscours européen. Si l'on prend par exemple la forme insertion, on remarque que ses co-occurrents ${ }^{27}$ statistiquement significatifs permettent de rendre compte de l'injonction selon laquelle l'université contemporaine doit permettre aux étudiants, à l'aide de stratégies d'orientation et d'aide à la réussite, d'obtenir un diplôme afin de s’insérer dans un milieu professionnel.

25 Cet interdiscours, entendu comme « l'ensemble des unités discursives (relevant de discours antérieurs du même genre, de discours contemporains d'autres genres, etc.) avec lesquelles un discours particulier entre en relation implicite ou explicite » (Maingueneau, 2009 : 77), a notamment été détaillé par Cussó (2008).

26 Le corpus de référence est Frantext. AnaText calcule le degré de spécificité à partir d'un ratio de fréquences des lemmes dans le corpus de travail et dans le corpus de référence.

27 En italique dans ce qui suit. 


\section{Des textes partiels et partiaux}

Décrire un objet sémiotique quel qu’il soit requiert une sélection réfléchie des aspects précis que l'on souhaite mettre en scène. Ce faisant, toute représentation descriptive sera nécessairement partielle. Dans le cas des universités, cela vaut pour le choix des différents thèmes évoqués : histoire de l'institution, présentation du campus, des formations, des différentes composantes (instituts, facultés, etc.), etc. Or, si l'on part du constat que de nombreux aspects sélectionnés sont aussi ceux retenus précisément par l'imaginaire néolibéral européen (caractère international de l'institution, relations avec le monde socioéconomique, excellence des enseignements, etc.), la description sera non seulement partielle mais également partiale : les textes sont vecteurs d'une idéologie. À ce niveau, par exemple, à travers la scène d'énonciation qu'ils construisent et plus précisément les arguments censés répondre à l'appel de lecteurs-modèle (Eco, 1985), tel lecteur empirique se verra interpellé2 en tant que stakeholder de l'université. Le discours promotionnel, qui demeure valorisant et neutre en apparence, témoigne ainsi, en filigrane, de la réalité d'une économie de la connaissance en devenir.

Nous suggérons que l'édification de cette mise en scène échappe en partie à la volonté des scripteurs empiriques : les régularités décrites, afférentes à la formation discursive néolibérale, montrent que ces textes, dispersés d'université en université, participent pourtant d'un même ordre du discours, de sorte qu'il paraît difficile que ces textes puissent dire autre chose que ce qu'ils disent. Dans une étude antérieure, nous écrivions :

Les régularités discursives détectées témoignent (...) de l'autonomie contrôlée à laquelle les universités sont actuellement assujetties. Si les textes européens garantissent et promeuvent l'autonomie des institutions académiques, celle-ci est néanmoins contrôlée par toute une série de dispositifs (évaluation des chercheurs, agences d'évaluation de la qualité de l'enseignement, etc.) qui rend cette autonomie paradoxale. (...) La dépendance discursive vis-à-vis de l'interdiscours européen, à travers un vocabulaire et des arguments qui ne lui sont plus spécifiques, est symptomatique de la dépendance des universités vis-à-vis de critères qui doivent désormais compter sur le "marché de l'enseignement supérieur ", où chaque établissement se doit d'exceller - et le faire savoir - dans certains domaines communs imposés par l'extérieur. (Gaspard, 2013b)

Cette perte d'autonomie institutionnelle s'accompagne donc d'une perte d'autonomie discursive, au profit de concessions envers un interdiscours néolibéral européen :

Les politiques de communication menées par les universités visent à la création d'une identité de marque spécifique. Elles visent la différenciation. Mais elles se heurtent, en amont, à la difficulté de développer une offre réellement distincte

28 C'est volontairement que nous faisons ici allusion à la célèbre formule d'Althusser : « Lidéologie interpelle les individus en sujets "(Althusser, $1978: 110$ ). 
tandis qu'en aval, les directives ministérielles et les injonctions aux changements sclérosent l'usage d'une expression créative. (Granget, 2009)

Cette perte d'autonomie rappelle ainsi l'illusion intrinsèque à toute idéologie telle que décrite chez Pêcheux : " alors qu'il croit parler et être à l'origine du sens qu'il produit, le locuteur est en réalité lui-même parlé, car sous l'emprise de déterminations [préconstruit et interdiscours] qu'il méconnaît et que l'examen des conditions de production du discours doit expliciter " (cité par Détrie, Siblot et al, 2001 : 147). Une étude ultérieure pourrait ainsi déterminer ces conditions de productions concrètes en analysant, dans une perspective ethnographique, la façon dont les textes promotionnels sont effectivement construits par les scripteurs universitaires en situation.

\section{Conclusion}

En introduction, nous proposions de montrer en quoi la pratique discursive consistant à promouvoir en ligne une université produit des textes numériques desquels il est possible de dégager des régularités qui témoignent de la présence d'un ordre du discours particulier s'y trouvant être manifesté. L'existence d'un discours promotionnel, tel qu'il se donne à voir dans ces textes, témoigne de l'adoption d'un impératif communicationnel au sein des services publics : cette « idéologie de la communication " n'est pas sans lien avec les processus de marchandisation et de néolibéralisation d'activités autrefois explicitement régulées par l'État. Ce faisant, les principes de communication institutionnelle, originellement associés à la sphère commerciale et entrepreneuriale et produits notamment par le méta-discours des professionnels, sont recontextualisés dans le secteur public, générant ainsi de nouvelles pratiques socio-discursives inédites, de sorte que le discours institutionnel se double dans certains cas, comme nous le soulignions plus haut, en discours promotionnel.

En outre, nous attirons l'attention sur les pratiques professionnelles associées aux différentes unités lexicales mobilisées dans le discours : excellence, partenariat, qualité, internationalisation, etc. Ces unités témoignent en effet de changements institutionnels bel et bien opérés par des actants réels. Par exemple, revendiquer une qualité des enseignements et de la recherche s'accompagne de nouvelles pratiques, inédites au sein des universités, telles que celles relatives à l'évaluation des chercheurs, aux relations avec les « agences d'évaluation de la qualité de l'enseignement ", à l'écriture de référentiels de compétences, etc. Toute pratique discursive s'accompagne ainsi de pratiques professionnelles de sorte qu'on ne peut postuler l'autonomie de discours qui s'opposeraient aux différents contextes des pratiques qui les génèrent. Si le discours promotionnel disséminé par les textes en ligne de présentation a des raisons d'être qui lui sont propres, il est également le reflet d'une pratique professionnelle. Les régularités discursives sont ainsi générées par la régularité de pratiques et leurs contraintes, qui, en retour, engendrent et consolident des régularités discursives. 


\section{BIBLIOGRAPHIE}

ACHARD P., "Analyse du discours et sociologie du langage ", Langage et société, $\mathrm{n}^{\circ} 37,1986$, p. 5-60.

ADAM J.-M., BONHOMME M., L'argumentation publicitaire : rhétorique de l'éloge et de la persuasion, Paris : Armand Colin, 2012.

ALTHUSSER L., "Idéologie et appareils idéologiques d'État (Notes pour une recherche) », in Positions (1964-1975), Althusser L., Paris : Les Éditions sociales, 1976, p. 67-125.

AMOSSY R., HERSCHBERG-PIERROT A., Stéréotypes et clichés : langue, discours, société. Paris : Armand Colin, 2007.

ASKEHAVE I., "The impact of marketization on higher education genres - the international student prospectus as a case in point ", Discourse Studies, vol. 9, n 6, 2007, p. 723-742.

BAKHTINE M., "Les genres du discours », in Esthétique de la création verbale, Bakhtine M., Paris : Gallimard, 1984, p. 263-308.

BARATS C., "Textes numériques ou sur le numérique: rhétorique de l'internationalisation et "attractivité" des universités parisiennes ", in Acteurs et contextes des discours universitaires, Defays J.-M., Englebert A. (Dir.), volume 2, Paris : L'Harmattan, 2009, p. 209-223.

BRUNOT I., CLEMENT P., LAVAL C., La grande mutation: néolibéralisme et éducation en Europe, Paris : Éditions Syllepse, 2010.

CHARAUDEAU P., Grammaire du sens et de l'expression, Paris : Hachette Éducation, 1992.

CUSSÓ R., "Quand la Commission européenne promeut la société de la connaissance ", Mots. Les langages du politique, ${ }^{\circ}$ 88, 2008, p. 39-52.

DARBELLAY F., Interdisciplinarité et transdisciplinarité en analyse des discours : complexité des textes, intertextualité et transtextualité, Genève : Slatkine, 2005.

DEFAYS J.-M., « Défense et illustration de l'analyse des discours universitaires », in Principes et typologie des discours universitaires, Defays J.-M., Englebert E. (Dir.), volume 1, Paris : L'Harmattan, 2009, p. 9-22.

DÉTRIE C., SIBLOT P., VERINE B., Termes et concepts pour l'analyse du discours: une approche praxématique, Paris : Honoré Champion, 2001.

ECO U., Lector in fabula. Le rôle du lecteur ou La coopération interprétative dans les textes narratifs, Paris : Le Livre de poche, 1985.

ENGWALL L., "Minerva and the Media. Universities Protecting and Promoting Themselves ", in Universities in Transition: Issues, Models, and Cases, Mazza C., Quattrone P., Riccaboni A. (Dir.), Cheltenham : Edward Elgar Publishing, 2008 p. 31-48.

FÈVRE-PERNET C., "Readymades linguistiques et genres promotionnels », Signes, Discours E̊ Sociétés, n 4, 2009, http://www.revue-signes.info/document.php?id=1317 (consulté le 10/07/13).

FOUCAULT M., L'archéologie du savoir, Paris : Gallimard, 2010.

GASPARD J., "Le discours promotionnel des universités : homogénéité dans la compétitivité ? ", Mots. Les langages du politique, n 102, 2013a, p. 53-66.

GASPARD J., « L'européanisation de l'Enseignement supérieur en Europe : une analyse du discours promotionnel en ligne des universités ", Éducation comparée, n ${ }^{\circ}$, 2013b, p. 127-147. 
GRANGET L., "Les universités en quête de prestige dans le grand jeu de la concurrence : le rôle de la communication marketing et l'impact des palmarès ", Communication Eं organisation, $\mathrm{n}^{\circ}$ 35, 2009, p. 149-157.

KRIEG-PLANQUE A., "Travailler les discours dans la pluridisciplinarité. Exemples d'une "manière de faire" en analyse du discours ", in Analyse du discours et sciences humaines et sociales, Bonnafous S., Temmar M. (Dir.), Paris : Ophrys, 2007, p. 57-71.

LE BART C., TEILLET P., « Erreur, lapsus, gaffes, fautes...Le discours politique comme genre ", in L'analyse de discours, Ringoot R. \& Robert-Demontrond P. (Dir.), Rennes : Apogée, 2004, p. 53-85.

MAINGUENEAU D., Genèses du discours, Bruxelles : Mardaga, 1984.

MAINGUENEAU D., Les termes clés de l'analyse du discours, Paris : Seuil, 2009.

MAINGUENEAU D., "Genres de discours et web : existe-t-il des genres web ? ", in Manuel d'analyse du web en Sciences humaines et sociales, BARATS C. (Dir.), Paris : Armand Colin, 2013, p. 74-92.

MILNER J. C., Introduction à une science du langage, Paris : Seuil, 1995.

MORPHEW C., HARTLEY M., "Mission statements: A thematic analysis of rhetoric across institutional type », The Journal of Higher Education, vol. 77, n 3, 2006, p. 456-471.

OGER C., OLLIVIER-YANNIV C., «Analyse du discours institutionnel et sociologie compréhensive : vers une anthropologie des discours institutionnels», Mots. Les langages du politique, $\mathrm{n}^{\circ}$ 71, 2003, p. 125-144.

OSMAN H., "Re-branding academic institutions with corporate advertising: a genre perspective ", Discourse E Communication, vol. 2, n 1, 2008, p. 57-77.

SAICHAIE K., Representation on college and university websites : an approach using critical discourse analysis, thèse de doctorat, University of Iowa : Iowa City, 2011.

SARFATI G.-E., Eléments d'analyse du discours, $2^{\mathrm{e}}$ édition, Paris : Armand Colin, 2005.

Résumé : Dans un environnement professionnel devenu concurrentiel, les universités produisent de nombreux textes de communication, prioritairement en ligne, parmi lesquels on retrouve les textes de présentation d'universités, emblématiques de ce discours promotionnel. À travers cette contribution, nous désirons présenter ces productions discursives spécifiques et plus précisément les régularités discursives (génériques et interdiscursives) qui s'y trouvent être disséminées, et dont nous suggérons qu'elles témoignent de l'actualisation d'un ordre du discours particulier. À partir d'un corpus issu de sites web d'universités francophones en Europe, nous désirons montrer que ces textes numériques, tout en décrivant l'institution de manière valorisante en vue de la promouvoir, sont nécessairement partiels mais également partiaux : apparentés à la formation discursive néolibérale liée aux politiques transnationales relatives à l'enseignement supérieur en Europe, ils sont alors vecteurs d'idéologie.

Mots clés : Analyse du discours, université, genre de discours, formation discursive, textométrie.

Abstract : In a highly competitive professional environment, universities produce a multitude of online promotional texts among which belong those presenting the institutional as a whole. With this contribution, we wish to describe these specific discursive productions and more 
precisely the (generic and interdiscursive) regularities which are being manifested in those texts and indicate the presence of a particular order of discourse. Based on a textual corpus built upon francophone university websites in Europe, we wish to show that these numeric texts, while describing the institution in a valorizing way in order to promote it, are also partial: belonging to a specific discursive formation linked to transnational policies regarding Higher Education in Europe, they are, upon everything, the vector of a neoliberal ideology.

Keywords : Discourse analysis, university - discourse genres, discursive formation, textometry. 